\title{
Anti-Dementia Agents are Partially Symptomatic Treatment and Partially Disease Modifying Treatment
}

Koji Hori $^{1^{*}}$, Kimiko Konishi ${ }^{1,2}$, Masayuki Tani ${ }^{3}$, Norihisa Akashi ${ }^{3}$, Yuka Kitajima $^{4}$ and Mitsugu Hachisu ${ }^{5}$

${ }^{1}$ Department of Psychiatry, Showa University Northern Yokohama Hospital, Kanagawa, Japan

${ }^{2}$ Tokyo Metropolitan Tobu Medical Center for Persons WITH Developmental/Multiple Disabilities, Tokyo, Japan

${ }^{3}$ Department of Psychiatry, Showa University Karasuyama Hospital, Tokyo, Japan

${ }^{4}$ Department of Anesthegiology, School of Medicine Juntendo University, Tokyo, Japan

${ }^{5}$ Department of Clinical Psychopharmacy, School of Pharmaceutical Sciences, Showa University, Tokyo, Japan

*Corresponding author: Koji Hori, Department of Psychiatry, Showa University Northern Yokohama Hospital, 35-1 Chigasakichuo, Tsuzukiku, Yokohama-City, Kanagawa, 224-8503, Japan, Tel: +81-45-949-7000; Fax: +81-45-949-7927; E-mail: kojihori@med.showa-u.ac.jp

Rec date: Mar 14, 2014, Acc date: Oct 18, 2014, Pub date: Oct 28, 2014

Copyright: ( 2014 Hori K, et al. This is an open-access article distributed under the terms of the Creative Commons Attribution License, which permits unrestricted use, distribution, and reproduction in any medium, provided the original author and source are credited.

\section{Short Commentary}

Antidementia agents, i.e., cholinesterase inhibitors (ChEIs) and Nmethyl-D-aspartate (NMDA) receptor (NMDA-R) antagonist are now considered to be symptomatic treatment. However, 4 ChEIs and 1 NMDA-R antagonist are proven to have neuroprotecting actions against amyloid pathology [1-4]. Amyloid has thought to have deteriorating actions to neurons especially cholinergic neurons (neurons those use acetylcholine (ACh) as neurotransmitter). However, reported that downregulation of ACh accelerated accumulation of amyloid, i.e., there were interactions between downregulation of $\mathrm{ACh}$ and accumulation of amyloid [5]. If so, downregulation of ACh (or hyperactivation of NMDA receptor) might be included in amyloidgenic process. Accordingly, antidementia agents might have not only symptomatic treatment property but also disease modifying treatment property. Moreover, there is needed the hypothesis that connects downregulation of $\mathrm{ACh}$ and accelerated accumulation of amyloid. In this article, we introduce our articles those showed endogenous hypothesis of anticholinergic activity (AA) in Alzheimer's disease (AD) and we speculate the disease modifying property in antidementia agents.

We reported that the relationships between AA using serum anticholinergic activity (SAA) and clinical symptoms in $\mathrm{AD}[6,7]$. Among 76 AD patients, 26 positive for SAA [SAA (+)] group had been prescribed a significantly higher number of psychotropic medications, had been diagnosed with a significantly more severe stage of $\mathrm{AD}$, had exhibited significantly lower cognitive functions, and had displayed significantly more severe behavior symptoms such as delusions, hallucinations, and diurnal rhythm disturbances. Logistic regression analysis revealed that there were significant correlations between SAA and the presence of delusion and diurnal rhythm disturbances. As for cognitive dysfunction, immediate memory and recall were significantly lower in the SAA (+) group than those in the SAA (-) group. These results were almost the same those of other reports. However, we showed a new finding in these two articles. The first new finding was the endogenous appearance of $\mathrm{AA}$ in $\mathrm{AD}$. We determined that AA was caused by psychotropic medications and led to behavioral symptoms. Moreover, we reasoned that because we generally prescribe psychotropic medicines for the clinical psychiatric symptoms of agitation and psychosis in $\mathrm{AD}$, cyclic relationships might exist among these three factors. We named this vicious cycle, the "vicious cycle of $\mathrm{AA}$ in $\mathrm{AD}$ (VCAA)". Because we generally prescribe psychotropic medicines for the clinical psychiatric symptoms of agitation and psychosis in $\mathrm{AD}$, endogenous AA may be observed [6,7].
Moreover, we speculated the reasons for the endogenous appearance of $\mathrm{AA}$ in $\mathrm{AD}$ and acceleration of $\mathrm{AD}$ pathology [8-10] by way of review of articles those with key words; inflammations, downregulations of ACh and AA because AA appears by way of inflammation with endogenous manners. Because ACh controls activity of inflammation in both central nervous system (CNS) and in peripheral tissues and $\mathrm{AD}$ is characterized by that cholinergic system is downregulated, inflammatory processes in both CNS and peripheral tissues might be caused by the downregulation of ACh and NMDA receptor expression is also upregulated by the downregulation of $\mathrm{ACh}$ which leads to hyperactivity of the inflammatory system. Cytokines that have AA might appear as a result of the inflammation. Therefore, we previously hypothesized that both AA in CNS (CAA) and peripheral tissue (serum anticholinergic activity; SAA) might appear endogenously in the moderate stage of $\mathrm{AD}$; the "endogenous anticholinergic hypothesis in $\mathrm{AD}$ " [8-10].

On the base of these speculations, because amyloid appears even in normal aged people and even in mild stage in $\mathrm{AD}$ [10], we also speculated that there might be at least three amyloid patterns in AD; normal pattern ( $\mathrm{N}$ pattern) which might be needed for normal aging, pathological pattern not related with downregulation of ACh (P1 pattern) and pathological pattern related with downregulation of $\mathrm{ACh}$ (P2 pattern) (Figure 1) [8]. In $\mathrm{AD}$ during mild cognitive impairment (MCI) and mild stage by $\mathrm{P} 1$ amyloid cognitive decline is slow and at moderate stage by $\mathrm{P} 1$ and $\mathrm{P} 2$ amyloid cognitive decline is rapid (Figure 1). Based on this, ACh upregulation and NMDA receptor downregulation may relate to both the symptoms in $\mathrm{AD}$ and the amyloid-producing process of the P2 pattern. Two AD pharmacotherapeutic options exist: prevention and treatment. ChEIs maintain normal ACh levels, prevents rapid neuron degeneration and delays the appearance of $\mathrm{P} 2$ pattern amyloid. This therefore delays the rapid progression of $\mathrm{AD}$ (Figure 2). NMDA receptor (NMDA-R) antagonists are then efficacious for decreasing the speed of $A D$ progression during the moderate stage (Figure 3 ). 


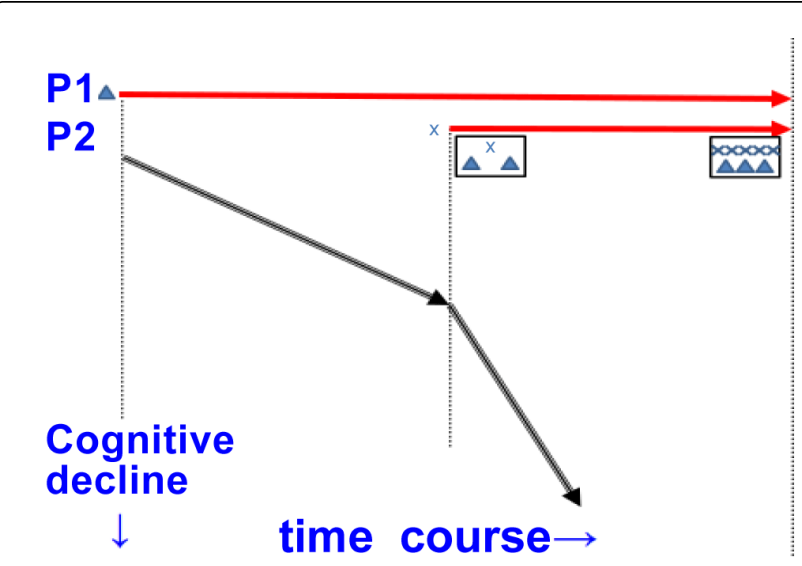

Figure 1: The normal patter amyloid ( $\mathrm{N}$ pattern amyloid) represents a physiological pattern, which is related to normal aging. The P1 pattern amyloid (pathological pattern amyloid not related with downregulation of $\mathrm{ACh}$ ) represents a pathological pattern unrelated to ACh downregulation and typically observed in MCI or mild AD. The P2 (pathological pattern amyloid related with downregulation of $\mathrm{ACh}$ ) pattern also represents a pathological pattern, and represents that which we postulate is related to the ACh downregulation observed in moderate $\mathrm{AD}$. In the brain P1 pattern and P2 pattern amyloids appear. This figure is from the article by Hori et al. [8] and partially exchanged.

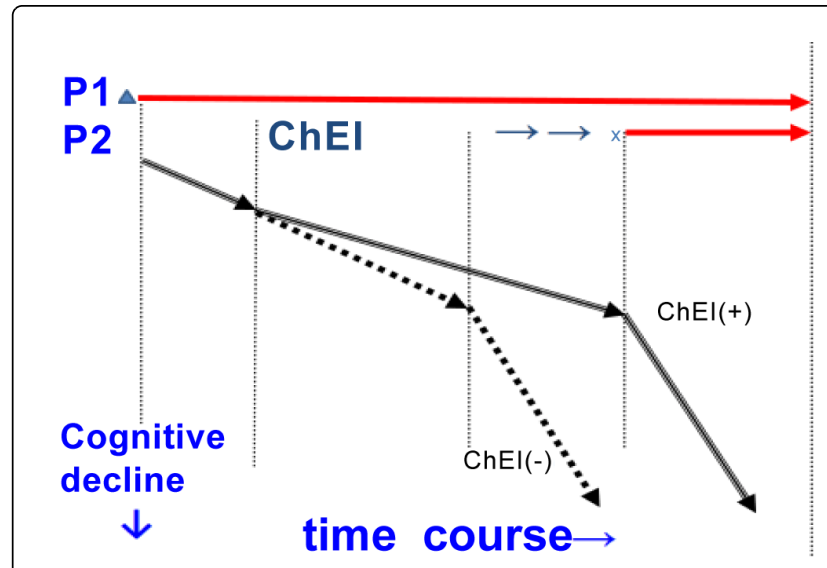

Figure 2: ChEIs maintain normal ACh levels, prevents rapid neuron degeneration and delays the appearance of $\mathrm{P} 2$ pattern amyloid. This therefore delays the rapid progression of $\mathrm{AD}$.

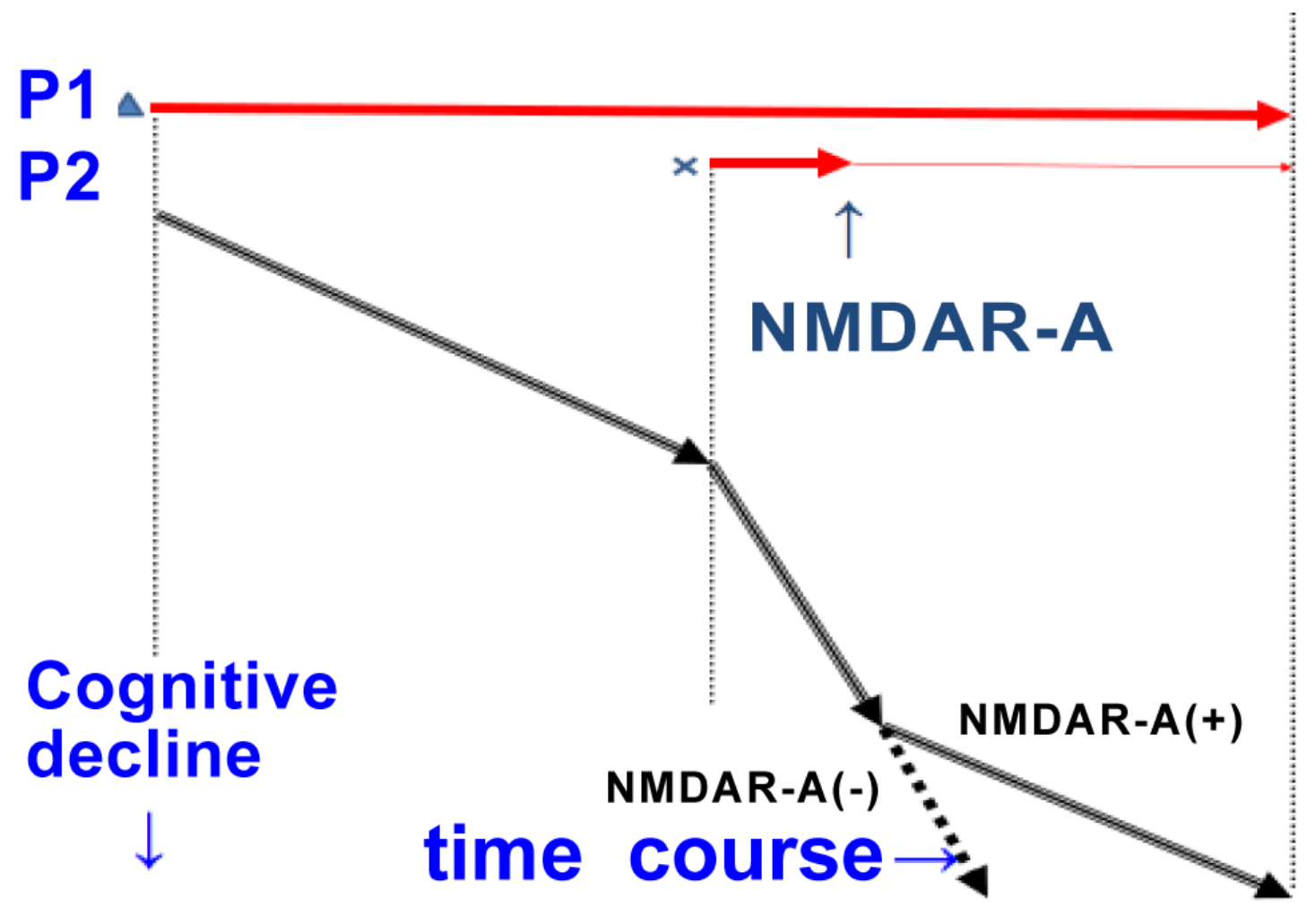

Figure 3: NMDAR-A is efficacious for decreasing the speed of AD progression during the moderate stage. 
On the basis of the above considerations, we also speculated that antidementia agents, i.e., cholinesterase inhibitors (ChEIs) and NMDA-R antagonist are partially symptomatic treatment (for P1 pattern amyloid) and partially disease modifying treatment (for P2 pattern amyloid).

We can explain other two facts from this speculation. This speculation may explain a limitation of the "amyloid vaccine" for AD. As previously mentioned, three amyloidogenic patterns may exist. If the $\mathrm{N}$ pattern amyloid is necessary for normal brain maturation, then P1 (and/or P2) amyloid patterns should be abolished.

Moreover, this speculation also explains why ChEIs can't prevent the conversion from MCI to mild dementia. We consider that we should investigate the mechanism underlying P1 pattern amyloid and amyloidogenesis. At present, there is no preventive therapy against the P1 pattern [10].

Although we consider that we should prove our hypothesis, AA may be a final common pathway in the amyloid-producing process, and may represent an interface between inflammation (downregulation of $\mathrm{ACh}$ ) and the amyloid-producing process [10].

Finally, we consider that if a treatment goes well, progress of $\mathrm{AD}$ is slow. In this case, hyperactivation of inflammatory system is prevented and $\mathrm{P} 2$ pattern amyloid could not appear. On the contrary P1 pattern amyloid appear continuously.

Therefore, not the rapid decline but the slow decline of cognitive function could occur even at moderate stage (Figure 4). From this point we should find the new pharmacological treatments for preventing hyperactivation of inflammatory system in order not to accelerate the speed of decline of cognitive function of AD.

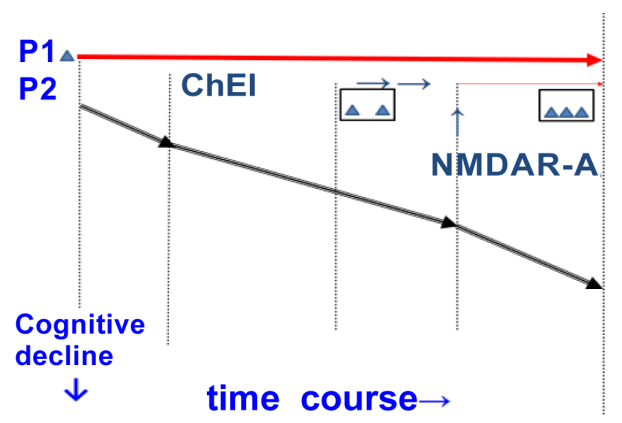

Figure 4: If a treatment goes well, progress of $\mathrm{AD}$ is slow. In this case, hyperactivation of inflammatory system is prevented and P2 pattern amyloid could not appear. On the contrary P1 pattern amyloid appear continuously. Therefore, not the rapid decline but the slow decline of cognitive function could occur even at moderate stage. In the brain P1 pattern amyloids appear. ACh: acetylcholine, AD: Alzheimer's disease, ChEI: cholinesterase inhibitor, MCI: mild cognitive impairment, NMDAR-A: N-methyl-D-aspartate receptor antagonist. Red arrows show the three patterns of amyloid. : P1 pattern amyloid, $\times$ : P2 pattern amyloid.

There may be a possibility that these hypothesis are over speculations. However, we constitute these hypotheses step by step. As showing first, we evaluating relationships between AA (or SAA) and clinical symptoms in $\mathrm{AD}$. Based on these results, we speculated the endogenous hypothesis in $\mathrm{AD}$. Then we reviewed the articles and speculated the reasons for endogenous appearance of $\mathrm{AA}$ in $\mathrm{AD}$, i.e., endogenous AA cascade in AD. As third step, we speculated three amyliodgenic patterns. Therefore, this hypothesis is not overspeculated. Moreover, there is needed the hypothesis that connects downregulation of $\mathrm{ACh}$ and accelerated accumulation of amyloid in order to elucidate AD pathology.

\section{Conflicts of interest}

Koji Hori received lecture fees from Eisai Co., Ltd.; Pfizer Japan Inc.; Novartis Pharma K.K.; Daiichi Sankyo Inc.; Ono Pharmaceutical Co., Ltd.; Janssen Pharmaceutical K.K.; Yoshitomi Yakuhin Co.; and Mitsubishi Tanabe Pharma Co. Mitsugu Hachisu received funding from Astellas Pharma Inc.; Meiji Seika Pharma Co., Ltd.; Dainippon Sumitomo Pharm Co., Ltd.; Eli Lilly Japan K.K.; and Shionogi \& Co., Ltd.. He also received lecture fees from Meiji Seika Pharma Co., Ltd. and Mitsubishi Tanabe Pharma Co.

\section{Acknowledgement}

The funding for this study was provided by Eisai Co., Ltd.; Daiichi Sankyo Inc.; and Ono Pharmaceutical Co., Ltd.

\section{References}

1. Takada Y, Yonezawa A, Kume T, Katsuki H, Kaneko S, et al. (2003) Nicotinic acetylcholine receptor-mediated neuroprotection by donepezil against glutamate neurotoxicity in rat cortical neurons. J Pharmacol Exp Ther 306: 772-777.

2. Takada-Takatori Y, Kume T, Sugimoto M, Katsuki H, Niidome T, et al. (2006) Neuroprotective effects of galanthamine and tacrine against glutamate neurotoxicity. Eur J Pharmacol 549: 19-26.

3. Nyakas C, Graine I, Halmy LG, Banerjee P, Luiten PG (2011) The basal forebrain cholinergic system in aging and dementia: rescuing cholinergic neurons from neurotoxic amyloid-beta42 with memantine. Behav Brain Res. 221: 594-603.

4. Bailey JA, Lahiri DK (2010) A novel effect of rivastigmine on presynaptic proteins and neuronal viability in a neurodegeneration model of fetal rat primary cortical cultures and its implication in Alzheimer's disease. J Neurochem 112: 843-853.

5. Jones CK, Brady AE, Davis AA, Xiang Z, Bubser M, et al (2008) Novel selective allosteric activator of the M1 muscarinic acetylcholine receptor regulates amyloid processing and produces antipsychotic-like activity in rats. J Neurosci. 28: 10422-10433.

6. Konishi K, Hori K, Uchida H, Watanabe K, Tominaga I, et al. (2010) Adverse effects of anticholinergic activity on cognitive functions in Alzheimer's disease. Psychogeriatrics 10: 34-38.

7. Hori K, Konishi K, Watanabe K, Uchida H, Tsuboi T, et al. (2011) Influence of anticholinergic activity in serum on clinical symptoms of Alzheimer's disease. Neuropsychobiology 63: 147-153.

8. Hori K, Konishi K, Akita R, Tani M, Tomioka H, et al. (2013) [Proposal of endogenous anticholinergic hypothesis in Alzheimer disease]. Nihon Shinkei Seishin Yakurigaku Zasshi 33: 117-126.

9. Hori K, Konishi K, Tani M, Tomioka H, Akita R, et al. (2014) Serum anticholinergic activity: A possible peripheral marker of the anticholinergic burden in the central nervous system in Alzheimer ${ }^{\text {tw }} s$ disease. Disease Markers 3: 459013.

10. Hori K, Konishi K, Tani M, et al. (2014) Why does the progression of Alzheimers $^{\text {Tw }}$ s disease accelerate? Ann Psychiatry Ment Health. 2: 1006. 